\title{
Psicooncología
}

ISSN: $1696-7240$

\section{Psicoterapia Centrada en el Sentido: Una revisión acerca de estrategias psicoterapéuticas para el tratamiento del paciente con cáncer}

\author{
Angélica Yolanda Bueno Bejarano Vale de Medeiros ${ }^{1, *}$; Eliane Ramos Pereira ${ }^{2}$; Rose Mary \\ Costa Rosa Andrade Silva ${ }^{3}$; Renata Carla Nencetti Pereira Rocha ${ }^{4}$
}

Recibido: 11 de abril de 2020 / 10 de agosto de 2020

Resumen: Objetivo: presentar las estrategias psicoterapéuticas utilizadas en el tratamiento de pacientes con cáncer, cuya finalidad sea trabajar el sentido de la vida. Método: Se realizó una revisión sistemática inscrita en el registro PROSPERO con el número CRD42019132385, siguiendo el método PRISMA, en las bases Lilacs, Medline, Scopus y Web of Science. Resultados: Se incluyeron 11 artículos publicados entre 2014 y 2020. La Psicoterapia Centrada en el Sentido (PCS) es la estrategia psicoterapéutica más utilizada y efectiva para ayudar al paciente acerca de su sentido de la vida. Discusión: Se desarrollaron tres categorías: 1. Encontrando el sentido de la vida por medio de la PCS; 2. Bienestar espiritual y psicológico de la PCS y 3. Terapias individuales, grupales y de pareja como estrategias eficaces de la PCS. Conclusiones: Este estudio señala que la PCS, además de ser beneficioso para los pacientes, puede servir como una alternativa de trabajo para el psicólogo principalmente en regiones de habla portuguesa donde no se encontraron estudios.

Palabras clave: Neoplasias, psicoterapia, espiritualidad, sentido de la vida.

\section{[en] Meaning centered psychotherapy: a review of psychotherapeutic strategies for the treatment of cancer patients}

\begin{abstract}
Objective: to present the psychotherapeutic strategies used in the treatment of cancer patients, whose purpose is to work in the meaning of life. Method: A systematic review was performed in the PROSPERO with the number CRD42019132385 registry, following the PRISMA method, based on Lilacs, Medline, Scopus and Web of Science. Results: 11 articles published between 2014
\end{abstract}

1 Angélica Yolanda Bueno Bejarano Vale de Medeiros. Programa Acadêmico em Ciências do Cuidado em Saúde, Escola de Enfermagem, Universidade Federal Fluminense, Niterói, Rio de Janeiro, Brasil

E-mail: angelicaflow@gmail.com

2 Eliane Ramos Pereira. Programa Acadêmico em Ciências do Cuidado em Saúde, Escola de Enfermagem, Universidade Federal Fluminense, Niterói, Rio de Janeiro, Brasil

E-mail: elianeramos.uff@gmail.com

3 Rose Mary Costa Rosa Andrade Silva. Programa Acadêmico em Ciências do Cuidado em Saúde, Escola de Enfermagem, Universidade Federal Fluminense, Niterói, Rio de Janeiro, Brasil

E-mail: roserosauff@gmail.com

4 Renata Carla Nencetti Pereira Rocha. Programa Acadêmico em Ciências do Cuidado em Saúde, Escola de Enfermagem, Universidade Federal Fluminense, Niterói, Rio de Janeiro, Brasil

E-mail: rnencetti@yahoo.com.br rasil

* Dirección de Correspondencia: Angélica Yolanda Bueno Bejarano Vale de Medeiros. Rua Dr. Celestino 74, 6to andar, Programa Acadêmico em Ciências do Cuidado em Saúde (PACCS). Bairro: Centro, CEP 24020-091, Niterói, RJ. Brasil. E-mail: angelicaflow@gmail.com

Fuente de Financiamiento y Agradecimientos: Coordenação de Aperfeiçoamento de Pessoal de Nível Superior (CAPES) 
and 2020 were included. Meaning-Centered Psychotherapy (MCP) is the most used and effective psychotherapeutic strategy to help the patient about their meaning in life. Discussion: Three categories have been developed: 1 . Find the meaning of life through MCP; 2. Spiritual and psychological wellbeing of the MCP and 3. Individual, group and couple therapies as effective strategies for the MCP. Conclusions: This study shows that MCP, in addition to being beneficial for patients, could serve as an alternative work for the psychologist, especially in regions of the Portuguese language where no studies were found.

Keywords: Neoplasms, psychotherapy, spirituality, meaning in life.

Sumario: 1. Introducción 2. Método 3. Resultados y discusión 4. Conclusiones 5. Referencias bibliográficas

Cómo citar: Bueno Bejarano Vale de Medeiros AY, Ramos Pereira E, Costa Rosa Andrade Silva RM, Nencetti Pereira Rocha RC. Psicoterapia Centrada en el Sentido: Una revisión acerca de estrategias psicoterapéuticas para el tratamiento del paciente con cáncer. Psicooncología 2020; 17: 311-333. doi: $10.5209 /$ psic. 68717

\section{Introducción}

El cáncer es considerado un problema de salud pública en el mundo, en Brasil, se esperan 625 mil nuevos casos para cada año del trienio 2020-2022 ${ }^{(1)}$. El sufrimiento de esta enfermedad tiene un impacto biopsicosocial y espiritual, surgiendo sentimientos de miedo a una muerte cercana, el sentimiento de pérdida del sentido de la vida, la amenaza y la incertidumbre ${ }^{(2)}$, desafiando a los pacientes a enfrentar situaciones de sufrimiento único ${ }^{(3)}$. Según Urrego et al. $(2015)^{(4)}$ entre las comorbilidades que pueden surgir, se encuentran las de aspecto psicológico, como la prevalencia de depresión en pacientes con cáncer entre 5 y 16\% para pacientes externos, entre 4 y $11 \%$ para pacientes hospitalizados y 7 a $49 \%$ para cuidados paliativos.

En el caso de los pacientes con cáncer avanzado, el sufrimiento está relacionado con problemas de pérdida de la esperanza, del bienestar espiritual, del sentido de la vida, aumentando la angustia existencial ${ }^{(3,-7)}$, esto debido a la posibilidad de una muerte próxima. En general, los cuidadores desconocen este tipo de angustia y en la mayoría de los casos estas preocupaciones están asociadas con cambios psicológicos y ajustes inadecuados, lo que aumenta el riesgo de depresión ${ }^{(3)}$.

Breitbart et al. $(2015)^{(5)}$ afirman que la evaluación y el tratamiento de trastornos psicológicos como la depresión y la ansiedad se reconocen como componentes críticos de la atención en oncología y cuidados paliativos, sin embargo, se utilizan pocas intervenciones que trabajen los temas espiritualidad y sentido de la vida, esto se puede atribuir a la falta de confianza de los médicos de salud mental para abordar dichos temas en el proceso de psicoterapia ${ }^{(8)}$.

Los estudios desarrollados hace más de una década por Breitbart $(2002)^{(9)}$ muestran que el nuevo enfoque clínico y de investigación en cuidados paliativos y atención a pacientes con cáncer es la espiritualidad, un término entendido desde una perspectiva existencial y filosófica que se refiere al conjunto de todas las emociones y convicciones de naturaleza no material, con la suposición de que hay más de lo que se puede percibir o entender en la vida, refiriéndose a cuestiones como el significado y el sentido de la vida, no limitado a ningún tipo específico de creencia o práctica religiosa ${ }^{(10-12)}$. 
La Organización Mundial de la Salud (OMS) ${ }^{(11)}$ incluyó la espiritualidad en el concepto de salud en 1998, como la dimensión que va más allá de la religiosidad, abordando la búsqueda y construcción del sentido y el propósito de la vida. Esta dimensión existencial también se incluyó en el concepto de cuidados paliativos como parte del tratamiento integral del paciente y su familia ${ }^{(4,11,13)}$.

Actualmente más del $40 \%$ de los pacientes con cáncer informan que desean ayuda de naturaleza espiritual para vencer los miedos, encontrar esperanza y especialmente encontrar el sentido de la vida ${ }^{(3)}$. El sentido de la vida (SV) se considera el motor de la existencia humana, y es posible atribuir sentido en cada situación e incluso en el sufrimiento $^{(12)}$. Este SV está relacionado con el valor que las personas atribuyen a las cosas, a las relaciones afectivas, y a sus propias vidas y, en el caso de los pacientes con cáncer, se refiere a las posibilidades de construir un sentido positivo para su condición de enfermedad ${ }^{(3)}$.

Esta atribución de sentido funciona como un amortiguador contra la angustia, ya que la evaluación de la situación es más positiva, proporcionándole una estructura psicológica que contribuye a enfrentar los procesos del tratamiento, ayudando en su adherencia ${ }^{(10)}$, aumenta la autoestima el optimismo y disminuye el sufrimiento ${ }^{(3)}$, reduce la ansiedad y el deseo de adelantar la muerte ${ }^{(5)}$.

Por lo tanto, trabajar en la espiritualidad desde la perspectiva del sentido de la vida ha sido un tema investigado en el campo de la salud durante más de una década, pero es poco conocido y practicado, especialmente en los países de América Latina, lo que puede convertirse en una estrategia efectiva, expandiendo el campo de la práctica multiprofesional en el área de oncología, con resultados favorables para pacientes, ya que la evidencia científica apunta a una mejora en el bienestar espiritual, una mejor adherencia al tratamiento y una mayor calidad de vida al final de la existencia ${ }^{(7,8,10)}$.

De acuerdo con lo anterior, el objetivo de este trabajo es presentar las estrategias psicoterapéuticas utilizadas en el tratamiento de pacientes con cáncer, cuya finalidad sea fortalecer o revelar el sentido de la vida.

\section{Método}

Se realizó una revisión de la literatura, previamente inscrita en el International prospective register of systematic reviews- PROSPERO con el número CRD42019132385, un registro internacional prospectivo de revisiones sistemáticas que incluye resultados relevantes para el paciente o la salud ${ }^{(14)}$. Esta revisión utilizó el Flow Diagram to Preferred Reporting Items for Systematic Reviews and Meta-Analyses- PRISMA, diagrama de flujo para elementos de informes preferidos para revisiones sistemáticas y metanálisis ${ }^{(15)}$.

Se adoptó la siguiente pregunta de investigación: ¿Cuáles son las estrategias psicoterapéuticas más utilizadas para el tratamiento de pacientes con cáncer, con el objetivo de fortalecer o revelar el sentido de la vida? La búsqueda de los estudios se realizó desde: a) Portal de la Biblioteca Virtual en Salud de Brasil (BVS), a través de las siguientes bases de datos: Literatura Latinoamericana y del Caribe en Ciencias de la Salud (LILACS), Medical Literature Analysis and Retrieval System on-line (MEDLINE) e Index Psicología- Periódicos técnico-Científicos y b) Plataforma: Coordenação de Aperfeiçoamento de Pessoal de Nível Superior 
(CAPES) de Brasil a través de las bases de datos: SciVerse SCOPUS y SciELO Citation Index (Web of Science).

Se llevaron a cabo dos estrategias de búsqueda, la primera combinó los descriptores y operadores booleanos: neoplasms AND psychotherapy AND spirituality, y la segunda estrategia los descriptores y operadores booleanos: neoplasms AND psychotherapy $A N D$ y la palabra clave Meaning en el idioma inglés para ampliar la búsqueda. Los términos utilizados se verificaron en los sitios web: Descriptores de ciencias de la salud (DeCS) y Medical Subject Headings (MeSH). Como criterio de inclusión, fueron seleccionados los artículos publicados entre los años 2014 y 2020 en español, portugués e inglés. Criterios de exclusión: cartas editoriales, artículos de revisión, sin acceso completo, artículos con diferentes propuestas terapéuticas para el propósito de esta revisión, población infantil, pacientes sobrevivientes o cuidadores familiares, la búsqueda fue realizada en 1 de Julio de 2020.

El resultado de las dos estrategias de búsqueda iniciales fueron 112 artículos, de los cuales la primera estrategia fue 35, $($ BVS Portal $=$ Bases: MEDLINE $=19$; LILACS $=2$; INDEXPSI $=0$ CAPES Portal $=$ Scopus $=13 ;$ Web of Sciencie SciELO Index $=1)$, de la segunda estrategia 77, (Portal BVS $=$ Bases: MEDILINE $=50$; LILACS $=1$; INDEXPSI $=0 ;$ Portal CAPES $=$ Scopus $=25$; Índice SciELO de Web of Science $=1)$. Después de la evaluación y selección utilizando los criterios de elegibilidad, se incluyeron 11 artículos, como se muestra en el Diagrama de flujo PRISMA, véase la figura 1.

Figura 1. Modelo PRISMA de la revisión sistemática de la Literatura.

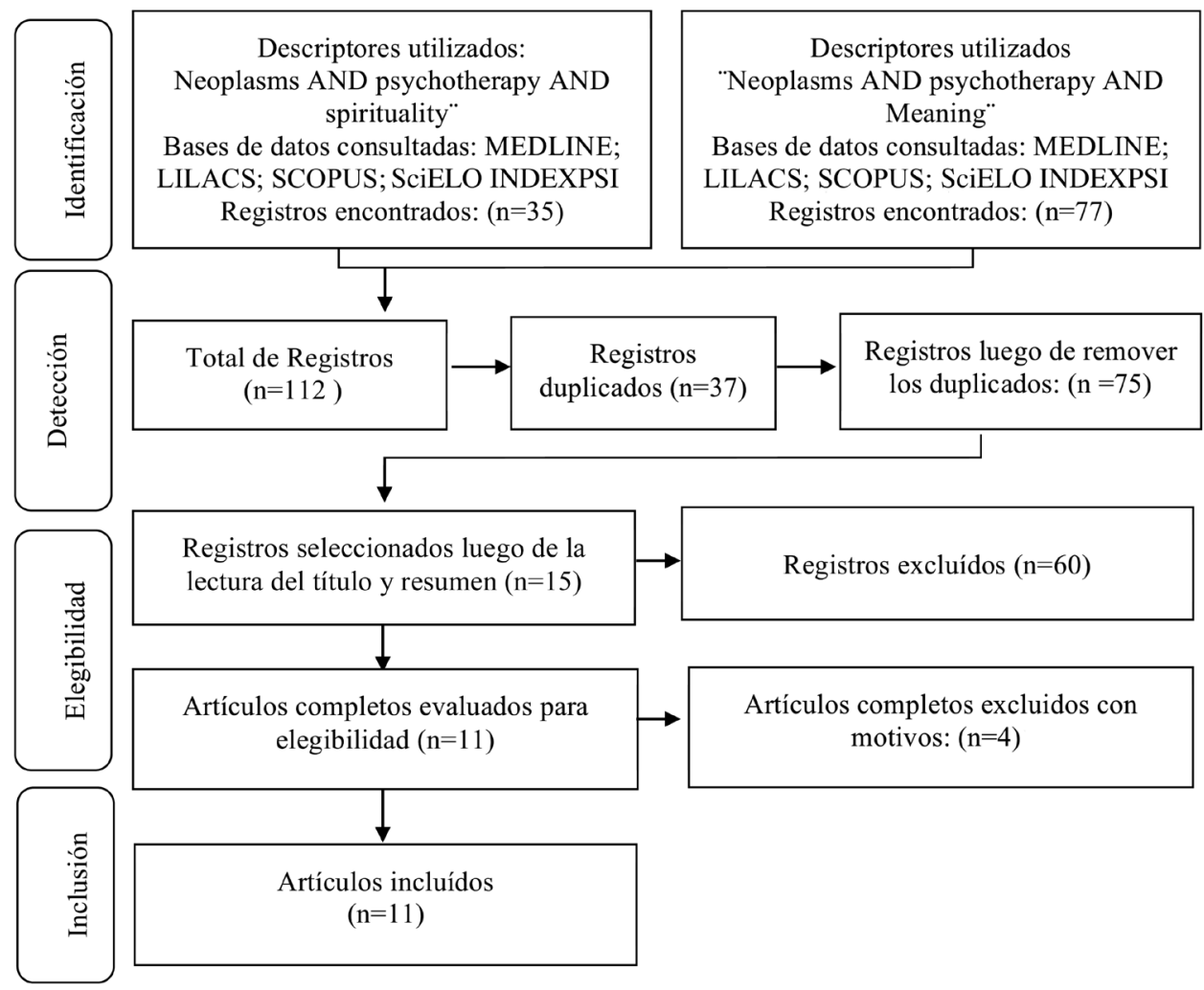




\section{Resultados y discusión}

En la tabla 1 se presenta la lista de los estudios seleccionados que incluyen la Psicoterapia Centrada en el Sentido como estrategia de intervención terapéutica que ayudan en el desvelar del sentido de la vida del paciente con cáncer.

Tabla 1. Artículos incluidos en la revisión de la Literatura

\begin{tabular}{|c|c|c|}
\hline Número & Nombre del Artículo & $\begin{array}{l}\text { Autor, } \\
\text { Año y País }\end{array}$ \\
\hline$[1]$ & $\begin{array}{l}\text { Meaning-Centered Psychotherapy for Latino Pa- } \\
\text { tients with Advanced Cancer: Cultural Adaptation } \\
\text { Process }\end{array}$ & $\begin{array}{l}\text { Costas-Muñiz et al. } \\
\text { (2020) } \\
\text { USA }\end{array}$ \\
\hline [2] & $\begin{array}{l}\text { Psychological intervention in patients with advanced } \\
\text { cancer at home through Individual Meaning-Cen- } \\
\text { tered Psychotherapy-Palliative Care: a pilot study }\end{array}$ & $\begin{array}{l}\text { Fraguell, Limonero \& } \\
\text { Gil } \\
(2020) \\
\text { España }\end{array}$ \\
\hline$[3]$ & $\begin{array}{l}\text { Individual meaning-centered psychotherapy for the } \\
\text { treatment of psychological and existential distress: } \\
\text { A randomized controlled trial in patients with ad- } \\
\text { vanced cancer }\end{array}$ & $\begin{array}{l}\text { Breitbart et al. } \\
(2018) \\
\text { USA }\end{array}$ \\
\hline$[4]$ & $\begin{array}{l}\text { Meaning-centered psychotherapy integrated with } \\
\text { elements of compassion: A pilot study to assess fea- } \\
\text { sibility and utility }\end{array}$ & $\begin{array}{l}\text { Gil et al. } \\
(2018) \\
\text { España }\end{array}$ \\
\hline$[5]$ & $\begin{array}{l}\text { Psychological aspects of meaning-centered group } \\
\text { psychotherapy: Spanish experience. }\end{array}$ & $\begin{array}{l}\text { Fraguell et al. } \\
(2018) \\
\text { España }\end{array}$ \\
\hline [6] & $\begin{array}{l}\text { Preliminary findings on the effectiveness of Mean- } \\
\text { ing-Centered Psychotherapy in Patients with pan- } \\
\text { creatobiliary cancer. }\end{array}$ & $\begin{array}{l}\text { Ryu et al. } \\
(2018) \\
\text { Corea del Sur }\end{array}$ \\
\hline$[7]$ & $\begin{array}{l}\text { Why is Meaning-Centered Group Psychotherapy } \\
\text { (MCGP) effective? Enhanced sense of meaning as } \\
\text { the mechanism of change for advanced cancer pa- } \\
\text { tients. }\end{array}$ & $\begin{array}{l}\text { Rosenfeld et al. } \\
(2017) \\
\text { USA }\end{array}$ \\
\hline$[8]$ & $\begin{array}{l}\text { Acceptability and Feasibility of a Meaning-Based } \\
\text { Intervention for Patients With Advanced Cancer and } \\
\text { Their Spouses: A Pilot Study. }\end{array}$ & $\begin{array}{l}\text { Wagner et al. } \\
(2016) \\
\text { USA }\end{array}$ \\
\hline [9] & $\begin{array}{l}\text { Meaning-centered group psychotherapy: an ef- } \\
\text { fective intervention for improving psychological } \\
\text { well-being in patients with advanced cancer. }\end{array}$ & $\begin{array}{l}\text { Breitbart et al. } \\
(2015) \\
\text { USA }\end{array}$ \\
\hline$[10]$ & $\begin{array}{l}\text { Development and preliminary testing of a mean- } \\
\text { ing-centered program for young adults with ad- } \\
\text { vanced-stage cancer. }\end{array}$ & $\begin{array}{l}\text { Kang \& Kim } \\
(2015) \\
\text { Corea del Sur }\end{array}$ \\
\hline$[11]$ & $\begin{array}{l}\text { Desarrollo de una intervención centrada en espiritu- } \\
\text { alidad en pacientes con cáncer. }\end{array}$ & $\begin{array}{l}\text { Urrego et al. } \\
(2015) \\
\text { Colombia }\end{array}$ \\
\hline
\end{tabular}


Todos los autores de los estudios seleccionados utilizaron como base para sus investigaciones el modelo de intervención llamado Psicoterapia Centrada en el Sentido (PCS) su nombre en inglés Meaning Centered Psychotherapy (MCP), desarrollado por el Dr. William Breitbart que comenzó con un grupo de investigación en 1995 en el Centro de Cáncer Memorial Hospital Sloan-Kettering en la ciudad de Nueva York, EE. UU., cuyo objetivo es estudiar las demandas de los pacientes con cáncer en una etapa avanzada, a saber: pérdida de bienestar espiritual, la necesidad de tener un sentido en la vida, sufrimiento existencial ${ }^{(5)}$ y la presencia de comorbilidades psicológicas como depresión, ansiedad y estrés ${ }^{(4)}$. Estimulados por la importancia del bienestar espiritual, su objetivo era ayudar al paciente en su experiencia de dar sentido a su vida, paz y propósito, incluso cuando está cerca del final de la vida ${ }^{(16,17,18)}$.

PCS fue desarrollada originalmente como una intervención grupal de 8 sesiones y fue posteriormente adaptada a un formato individualizado ${ }^{(19)}$.Los artículos incluidos y sus características varían según su intención de investigación con PCS, ya sea para probar su efectividad $[3,6,7,9]$, adaptación cultural y nuevos resultados $[1,5]$ o desarrollar un estudio piloto $[2,4,8,10,11]$. Los once estudios tienen como objetivo principal ayudar al paciente en la búsqueda del sentido de la vida, ocho estudios centraron en la atención de pacientes con cáncer avanzado, dos estudios con pacientes en cuidados paliativos y un estudio con pacientes diagnosticados con cáncer pancreatobiliar que se considera que tienen un peor pronóstico. Todos los artículos utilizaron el método de intervención, adoptando los siguientes diseños metodológicos: controlado y aleatorizado (4), mixto (2) e intervención en sí (5). Entre estos estudios, se encontraron cuatro tipos de intervención de PCS, basados en la propuesta de Breitbart: terapia individual con siete sesiones (4), terapia individual para pacientes en cuidados paliativos de tres sesiones (2) grupal (4) y de pareja (1). Los datos sintetizados y el análisis de los artículos incluidos se muestran en la tabla 2.

Como una forma de analizar y interpretar esta revisión, se desarrollaron tres categorías a ser discutidas a continuación: 1. Encontrando el sentido de la vida a través de la PCS, 2. Bienestar espiritual y psicológico de la PCS y 3. Terapias individuales, grupo y pareja como estrategias PCS efectivas. 


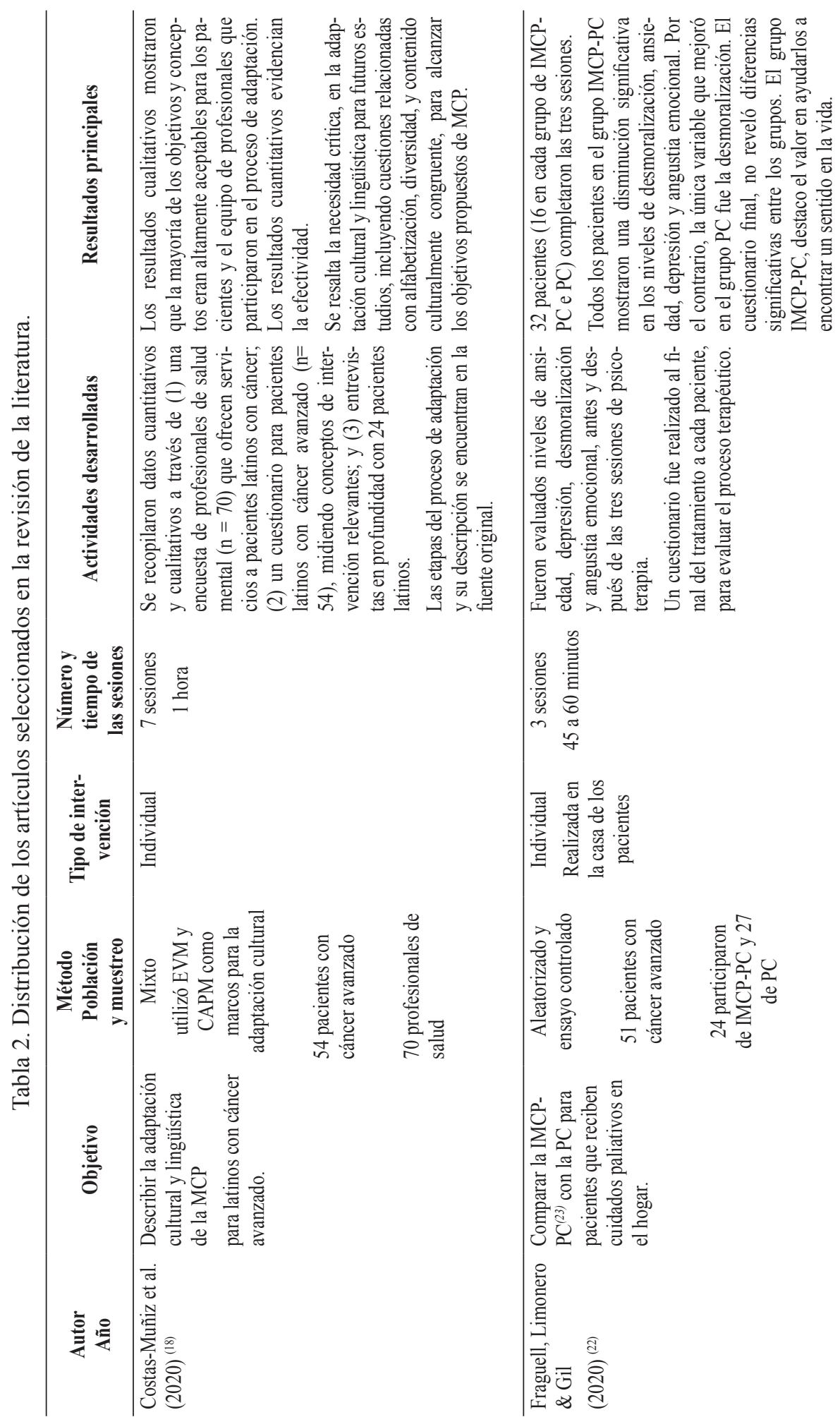




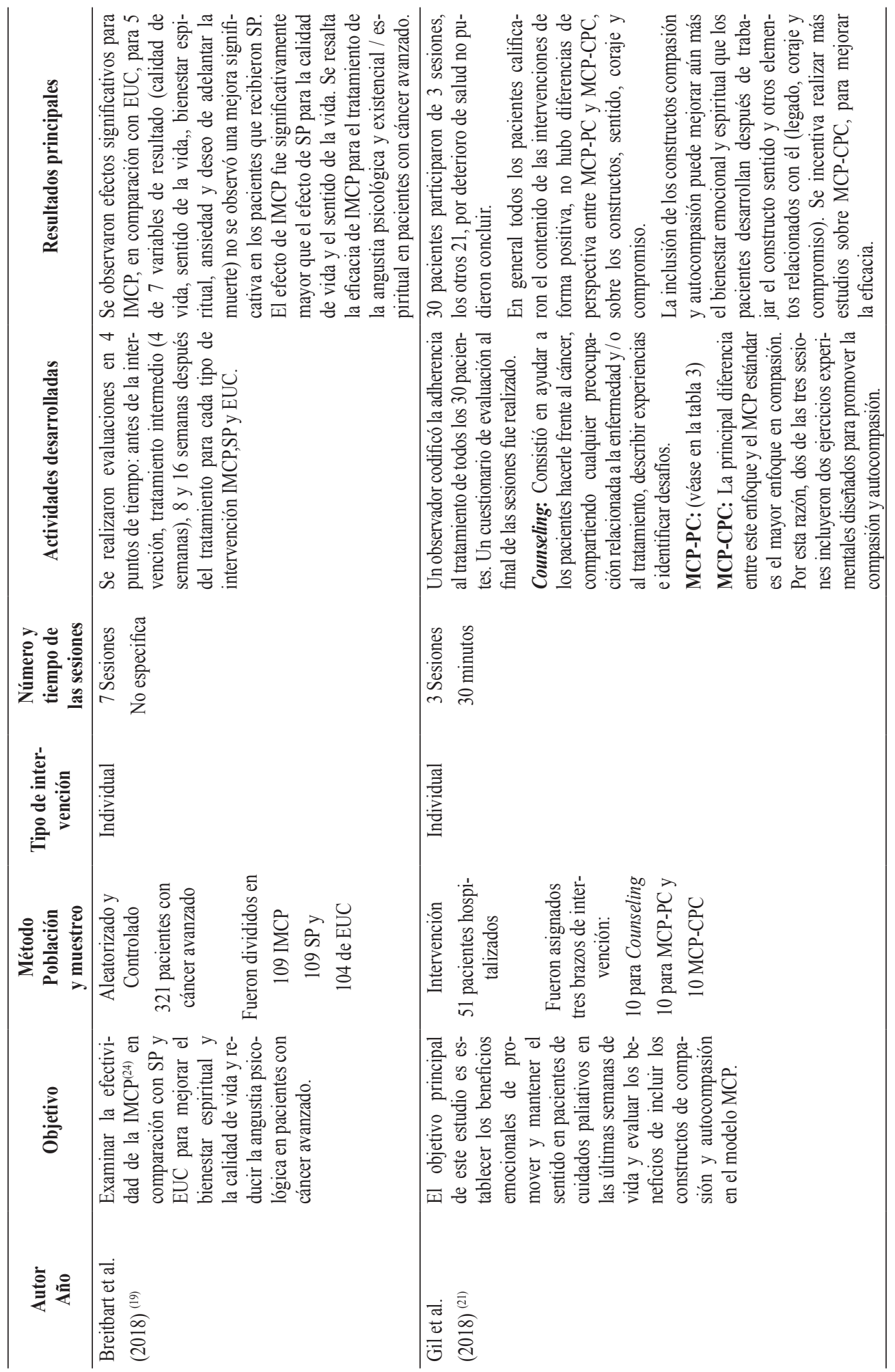



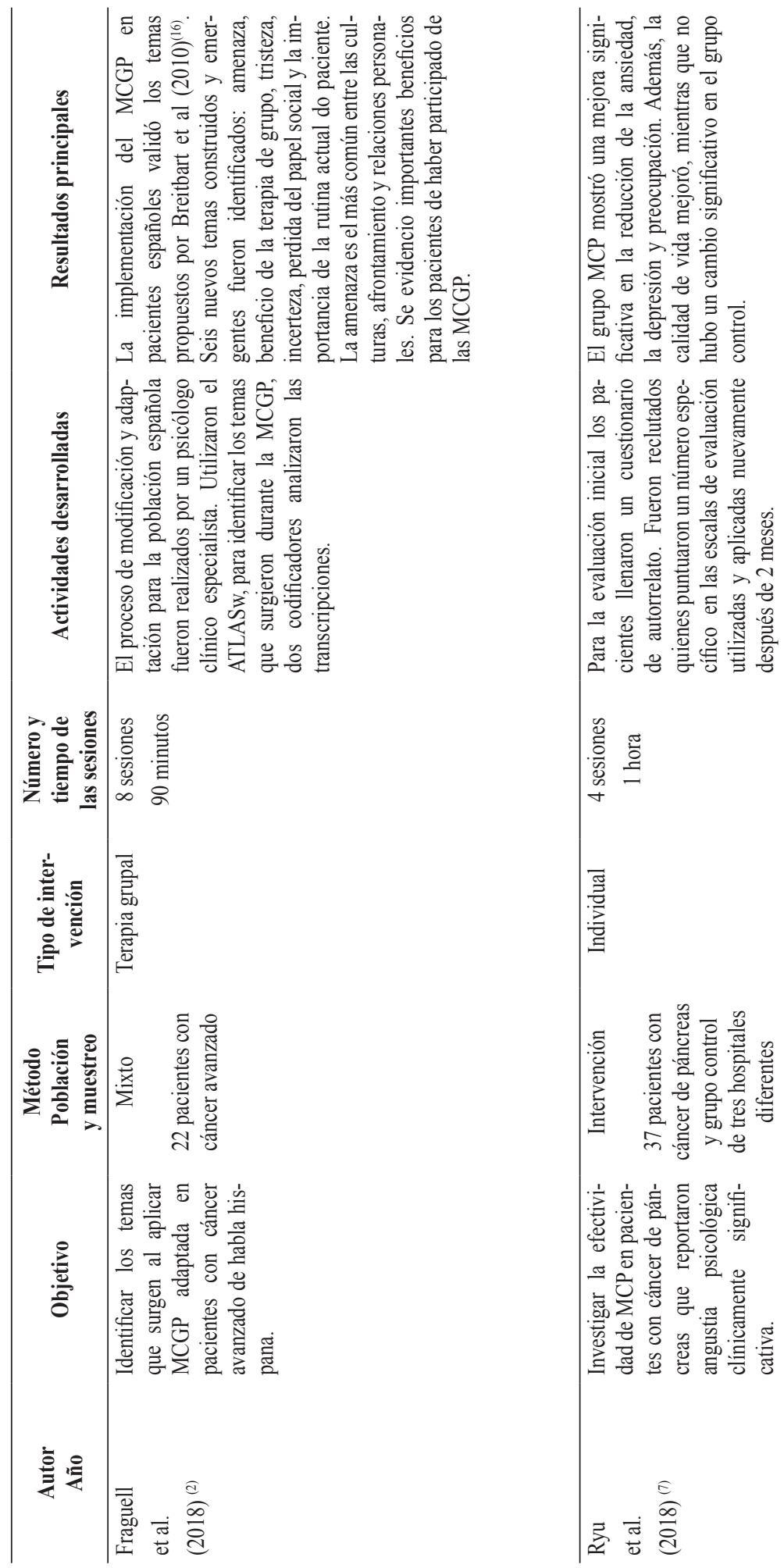


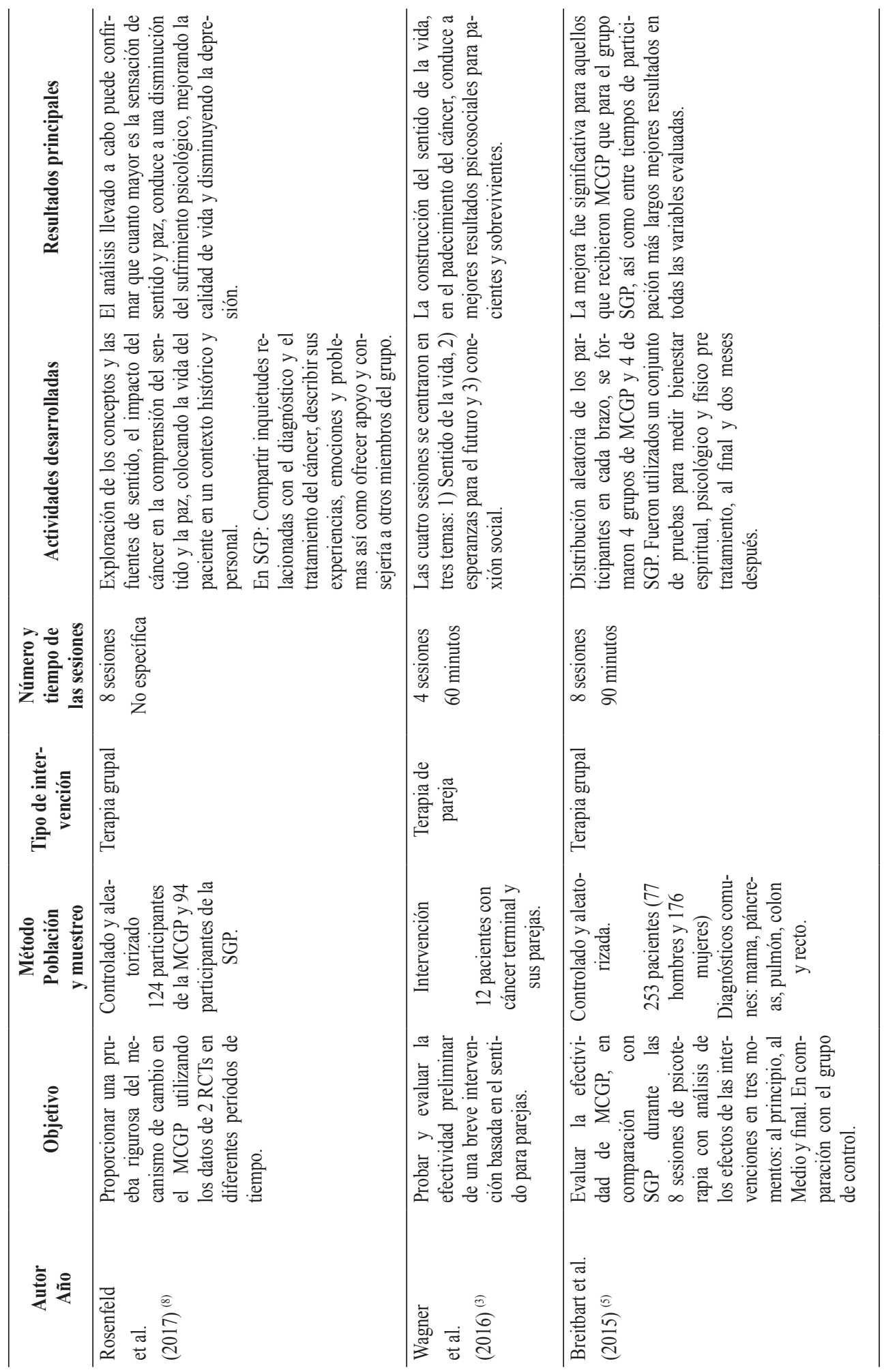



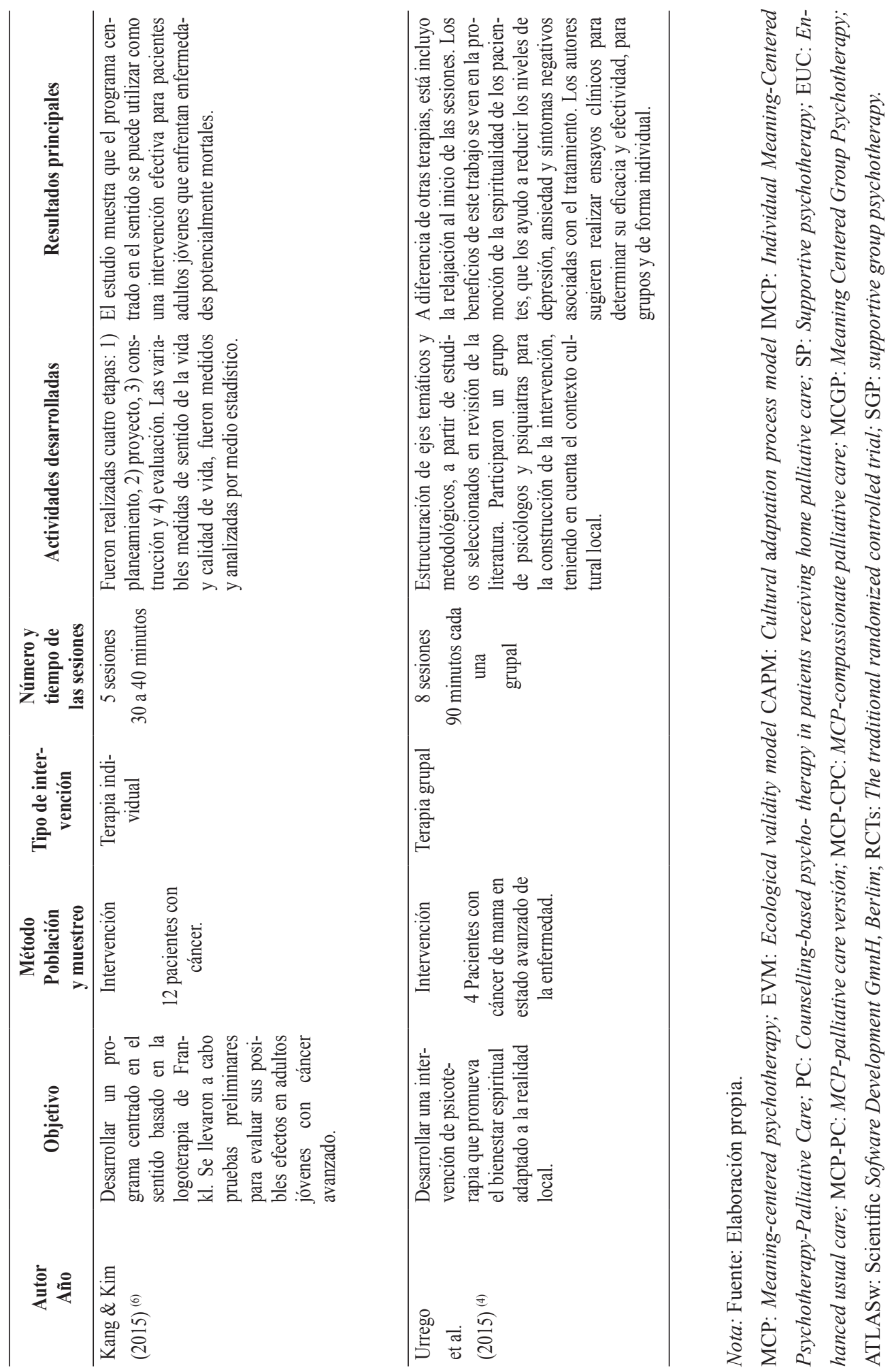


\section{A. Encontrando sentido de la vida a través de la Psicoterapia Centrada en el Sentido-PCS}

La PCS se basa teóricamente en la Logoterapia del Psiquiatra e Filosofo Dr. Viktor Emil Frankl (1905-1997) ${ }^{(2,3,4,5,5,6,7,8,16,18,19,21,22)}$, cuya teoría propone que es posible atribuir un sentido a la vida, en el sufrimiento e incluso minutos antes de la muerte. Para Frankl ${ }^{(12)}$ el hombre nace con una voluntad de sentido, una necesidad de buscar y encontrar una misión a ser cumplida en la existencia y afirmó que aunque el cuerpo esté enfermo, el espíritu del hombre nunca se enferma, puesto que existen las capacidades de superación, el potencial humano para tomar decisiones positivas frente al sufrimiento.

La filosofía de Frankl ${ }^{(12)}$ afirma que la espiritualidad es una fuente de SV y existen formas de encontrar, fortalecer o revelar este SV, por medio del desarrollo de valores. Los valores son una construcción cognitiva de lo que es más importante para las personas, lo más valioso y que moviliza el deseo de vivir. Los tres valores propuestos por Frankl ${ }^{(12)}$ son: 1. Creación; 2. Experiencia y 3. Actitud. Los de creación son todas las posibilidades de realizar, desarrollar y hacer algún trabajo, es lo que se da al mundo, al otro e para los otros. Los valores de experiencia, se refieren a las vivencias que traen buenos recuerdos, el aprendizaje, las relaciones, las conexiones con otros, con el mundo, con la naturaleza, el amor, el arte y el humor. Los valores de actitud, por otro lado, son aquellos que, a través de la capacidad interna de libertad de elección y responsabilidad frente a las condiciones de la vida, la persona puede actuar de manera apropiada delante a los desafíos de la existencia, es la capacidad de aprender del sufrimiento y verlo de una manera positiva ${ }^{(12)}$.

La PCS fue estructurada de tal manera que el paciente logre construir su sentido de la vida durante las sesiones, ya que trabaja con las fuentes del sentido de creación, experiencia y actitud. La tabla 3. muestra las características de las intervenciones utilizadas por cada estudio.

Tabla 3. Principales características de las intervenciones.

\begin{tabular}{|c|c|c|c|c|}
\hline Artículo & Objetivos & $\begin{array}{c}N^{0} \\
\text { Sesión }\end{array}$ & Temas & $\begin{array}{l}\text { Método Educativo } \\
\text { Aplicado }\end{array}$ \\
\hline \multirow{9}{*}{$\begin{array}{l}\text { Costas-Muñiz } \\
\text { et al. }(2020)^{(18)}\end{array}$} & \multirow{9}{*}{$\begin{array}{l}\text { Promover el aprendi- } \\
\text { zaje y el uso de fuentes } \\
\text { de sentido como recur- } \\
\text { sos para hacer frente al } \\
\text { cáncer avanzado según } \\
\text { MCP por medio de si- } \\
\text { ete sesiones de terapia, } \\
\text { en el modelo individual } \\
(\mathrm{IMCP})^{(24)}\end{array}$} & 1 & Conceptos y fuentes de sentido & $\begin{array}{l}\text { Introducción } \\
\text { Ejercicio experimental }\end{array}$ \\
\hline & & 2 & $\begin{array}{l}\text { Identidad y sentido, antes y } \\
\text { después del diagnóstico de cáncer }\end{array}$ & $\begin{array}{l}\text { Discusión } \\
\text { Ejercicio experimental }\end{array}$ \\
\hline & & 3 & $\begin{array}{l}\text { Fuentes históricas de sentido } \\
\text { (pasado legado, presente y legado } \\
\text { futuro) }\end{array}$ & $\begin{array}{l}\text { Discusión } \\
\text { Ejercicio experimental }\end{array}$ \\
\hline & & 4 & $\begin{array}{l}\text { Fuentes actitudinales de } \\
\text { sentido: Encuentro con la vida y } \\
\text { limitaciones }\end{array}$ & $\begin{array}{l}\text { Discusión } \\
\text { Ejercicio experimental }\end{array}$ \\
\hline & & \multirow{2}{*}{5} & \multirow{2}{*}{$\begin{array}{l}\text { Fuentes creativas de sentido: } \\
\text { Involucrarse en la vida plenamente }\end{array}$} & Discusión \\
\hline & & & & Ejercicio experimental \\
\hline & & \multirow{2}{*}{6} & \multirow{2}{*}{$\begin{array}{l}\text { Fuentes experimentales de sentido: } \\
\text { conexión con la vida }\end{array}$} & Discusión \\
\hline & & & & Ejercicio experimental \\
\hline & & 7 & $\begin{array}{l}\text { Transiciones: reflexiones y } \\
\text { esperanzas para el futuro }\end{array}$ & $\begin{array}{l}\text { Revisión, reflexión y } \\
\text { cierre. }\end{array}$ \\
\hline
\end{tabular}




\begin{tabular}{|c|c|c|c|c|}
\hline Artículo & Objetivos & $\begin{array}{c}\mathbf{N}^{0} \\
\text { Sesión }\end{array}$ & Temas & $\begin{array}{c}\text { Método Educativo } \\
\text { Aplicado }\end{array}$ \\
\hline \multirow{3}{*}{$\begin{array}{l}\text { Fraguell, } \\
\text { Limonero \& } \\
\text { Gil }(2020)^{(22)}\end{array}$} & $\begin{array}{l}\text { Cada sesión tuvo sus } \\
\text { objetivos: En la prime- } \\
\text { ra: obtener información } \\
\text { sobre la autoconciencia } \\
\text { de los pacientes y su } \\
\text { sentido de vida. }\end{array}$ & 1 & $\begin{array}{l}\text { Momentos importantes antes y des- } \\
\text { pués del diagnóstico, y los aspectos } \\
\text { que tiene influido en sus valores y } \\
\text { su propia identidad. } \\
\text { Al final de la sesión, se les anima a } \\
\text { explicar su historia a un ser querido } \\
\text { o amigo. }\end{array}$ & $\begin{array}{l}\text { Discusión, reflexión. } \\
\text { Ejercicio de conexión }\end{array}$ \\
\hline & $\begin{array}{l}\text { En la segunda: Se cen- } \\
\text { tra en las fuentes de } \\
\text { sentido: experiencia: } \\
\text { (amor, belleza y hu- } \\
\text { mor); creativo: (roles y } \\
\text { tareas) historia (legado) } \\
\text { y actitud (frente a las } \\
\text { limitaciones de la vida) }\end{array}$ & 2 & $\begin{array}{l}\text { Fuentes de sentido presentes en sus } \\
\text { vidas, a pesar de las limitaciones im- } \\
\text { puestas por la enfermedad. La sesión } \\
\text { finaliza con un ejercicio de reflexión } \\
\text { sobre sus fuentes de sentido y como } \\
\text { esto los hace sentir. }\end{array}$ & $\begin{array}{l}\text { Discusión y } \\
\text { Ejercicio de reflexión }\end{array}$ \\
\hline & $\begin{array}{l}\text { La tercera: ayudar a los } \\
\text { participantes a encon- } \\
\text { trar sentido a través del } \\
\text { coraje y el compromi- } \\
\text { so, de vivir su propio } \\
\text { legado y al encontrar } \\
\text { una sensación de paz. } \\
\end{array}$ & 3 & $\begin{array}{l}\text { Identificar experiencias pasadas de } \\
\text { coraje e describir sus cambios en } \\
\text { el contexto de su enfermedad. Los } \\
\text { participantes son invitados a hablar } \\
\text { sobre el legado que esperan dejar } \\
\text { en este mundo. }\end{array}$ & $\begin{array}{l}\text { Discusión y } \\
\text { Ejercicio de reflexión } \\
\text { Evaluar la experiencia } \\
\text { terapéutica y el sentido } \\
\text { para ellos. }\end{array}$ \\
\hline \multirow[t]{2}{*}{$\begin{array}{l}\text { Ryu et al. } \\
(2018)^{(7)}\end{array}$} & $\begin{array}{l}\text { Se definieron objeti- } \\
\text { vos para cada sesión: } \\
\text { Sesión uno: 1) Se pre- } \\
\text { parando para aceptar el } \\
\text { cáncer, } 2 \text { ) Observar y } \\
\text { controlar el estrés, } 3 \text { ) } \\
\text { Escribir una lista de } \\
\text { actividades que ofrecen } \\
\text { consuelo y alegría y } 4 \text { ) } \\
\text { Encontrar recursos . }\end{array}$ & 1 & $\begin{array}{l}\text { Aceptación de cáncer } \\
\text { Manejo del estrés }\end{array}$ & $\begin{array}{l}\text { Actividades de la } \\
\text { sesión } \\
\text { Actividades para la } \\
\text { casa }\end{array}$ \\
\hline & $\begin{array}{l}\text { Sesión dos: 1) Com- } \\
\text { prender el estilo de } \\
\text { afrontamiento, } \\
\text { 2) Aprender otras } \\
\text { estrategias de afronta- } \\
\text { miento, } \\
\text { 3) Observar y controlar } \\
\text { la ira. }\end{array}$ & 2 & $\begin{array}{l}\text { Aprendizaje de habilidades de } \\
\text { afrontamiento } \\
\text { Manejo de la ira }\end{array}$ & $\begin{array}{l}\text { Actividades de la } \\
\text { sesión } \\
\text { Actividades para la } \\
\text { casa }\end{array}$ \\
\hline
\end{tabular}

Sesión tres: 1) Introducción y visión general,

Reflexiones

2) Identidad antes $y$ después del diagnóstico 3 de cáncer y

Conceptos y fuentes de sentido. Cáncer y sentido.
Actividades de la sesión

Actividades para la casa.

3) La vida como un legado vivo (pasado, presente y vida). 


\begin{tabular}{|c|c|c|c|c|}
\hline Artículo & Objetivos & $\begin{array}{l}\mathbf{N}^{0} \\
\text { Sesión }\end{array}$ & Temas & $\begin{array}{l}\text { Método Educativo } \\
\text { Aplicado }\end{array}$ \\
\hline & $\begin{array}{l}\text { Sesión cuatro: 1) En- } \\
\text { contrar las limitaciones } \\
\text { de la vida, }\end{array}$ & & & \\
\hline & $\begin{array}{l}\text { 2) Involucrarse en la } \\
\text { vida a través de la crea- } \\
\text { tividad y las esperanzas } \\
\text { para el futuro y }\end{array}$ & 4 & $\begin{array}{l}\text { Fuentes de sentido: actitudinal, cre- } \\
\text { ativo y experiencial. }\end{array}$ & Reflexión y Discusión \\
\hline & $\begin{array}{l}\text { 3) Revisar las fuentes } \\
\text { de sentido y esperanzas } \\
\text { para el futuro. }\end{array}$ & & & \\
\hline \multirow{4}{*}{$\begin{array}{l}\text { Breitbart et al. } \\
(2015)^{(5)}\end{array}$} & \multirow[b]{2}{*}{$\begin{array}{l}\text { Se propusieron seis } \\
\text { objetivos a lo largo del } \\
\text { programa: }\end{array}$} & \multirow[b]{2}{*}{1} & \multirow[b]{2}{*}{ Conceptos y fuentes de sentido. } & $\begin{array}{l}\text { Presentación del obje- } \\
\text { tivo de la intervención. }\end{array}$ \\
\hline & & & & $\begin{array}{l}\text { Actividades que fo- } \\
\text { mentan la búsqueda del } \\
\text { sentido de la vida. }\end{array}$ \\
\hline & $\begin{array}{l}\text { Primero: Animar a los } \\
\text { pacientes con cáncer } \\
\text { a buscar sentido en } \\
\text { sus vidas; }\end{array}$ & 2 & Cáncer y sentido & $\begin{array}{l}\text { Actividades de pregun- } \\
\text { tas y respuestas } \\
\text { Reflexión y discusión }\end{array}$ \\
\hline & $\begin{array}{l}\text { sus vidas; } \\
\text { Segundo: A estimular la }\end{array}$ & 3 & $\begin{array}{l}\text { Fuentes históricas de sentido: lega- } \\
\text { do (pasado) }\end{array}$ & Reflexión y discusión \\
\hline \multirow[t]{6}{*}{$\begin{array}{l}\text { Rosenfeld et } \\
\text { al. }(2017)^{(8)}\end{array}$} & $\begin{array}{l}\text { trascendencia; } \\
\text { Tercero: La resiliencia; }\end{array}$ & 4 & $\begin{array}{l}\text { Fuentes históricas de sentido: lega- } \\
\text { do (presente y futuro) }\end{array}$ & Reflexión y discusión \\
\hline & $\begin{array}{l}\text { Cuarto: Integrar el di- } \\
\text { agnóstico de cáncer en }\end{array}$ & 5 & $\begin{array}{l}\text { Fuentes actitudinales de sentido: } \\
\text { Animando a pesar de las limitaci- }\end{array}$ & $\begin{array}{l}\text { Actividades de pregun- } \\
\text { tas y respuestas }\end{array}$ \\
\hline & la vida del paciente; & & ones de la vida & Reflexión y discusión \\
\hline & $\begin{array}{l}\text { Quinto: Expresar senti- } \\
\text { mientos y emociones y }\end{array}$ & 6 & $\begin{array}{l}\text { Fuentes creativas de sentido: creati- } \\
\text { vidad y responsabilidad }\end{array}$ & Reflexión y discusión \\
\hline & \multirow{2}{*}{$\begin{array}{l}\text { Sexto: Mejorar la adap- } \\
\text { tación psicológica, dan- } \\
\text { do sentido a la vida. }{ }^{(17)}\end{array}$} & 7 & $\begin{array}{l}\text { Fuentes experimentales de sentido: } \\
\text { Naturaleza, Arte y Humor. }\end{array}$ & Reflexión y discusión \\
\hline & & 8 & $\begin{array}{l}\text { Final: Decir adiós y esperanza para } \\
\text { el futuro. }\end{array}$ & $\begin{array}{l}\text { Evaluación y mante- } \\
\text { nimiento de posibles } \\
\text { mejoras después de } \\
\text { las terapias. }\end{array}$ \\
\hline
\end{tabular}

El objetivo con las intervenciones fueron: Aumentar el sentido de la vida y el sentido de la trascendencia, determinar los deseos y esperanzas, y ayudar a

Wagner et al. los pacientes y parejas a $(2016)^{(3)} \quad$ comunicarse más abiertamente sobre la muerte y el morir. Tres temas se centraron en las intervenciones: 1) sentido Discusión y reflexión.

Recordar y reflexionar sobre los eventos de la vida juntos. Cada pa1 reja recibió hojas de tareas para la próxima sesión, identificando sus deseos futuros.
Breve entrevista semiestructurada para evaluar la historia actual y pasada de problemas de salud mental.

Actividades para la casa. en la vida, 2) esperanzas para el futuro y 3 ) conexión social. 


\begin{tabular}{|c|c|c|c|c|}
\hline Artículo & Objetivos & $\begin{array}{c}\mathbf{N}^{0} \\
\text { Sesión }\end{array}$ & Temas & $\begin{array}{c}\text { Método Educativo } \\
\text { Aplicado }\end{array}$ \\
\hline \multirow{7}{*}{$\begin{array}{l}\text { Wagner et al. } \\
(2016)^{(3)}\end{array}$} & & & Revisión de tareas & \\
\hline & & & $\begin{array}{l}\text { Discutir los valores y deseos de } \\
\text { cada pareja como enfoques de fin } \\
\text { de vida. }\end{array}$ & $\begin{array}{l}\text { Revisando la actividad } \\
\text { de casa }\end{array}$ \\
\hline & & 2 & Completaron el juego de cartas Go & Actividad lúdica \\
\hline & & & Wish. & Actividad para la \\
\hline & & & $\begin{array}{l}\text { Folletos de reflexión y preparación } \\
\text { para la próxima sesión. }\end{array}$ & \\
\hline & & 3 & $\begin{array}{l}\text { Las parejas describieron momen- } \\
\text { tos significativos y cómo el cáncer } \\
\text { afectó ese sentido. }\end{array}$ & $\begin{array}{l}\text { Reflexión empática y } \\
\text { Discusión. }\end{array}$ \\
\hline & & 4 & $\begin{array}{l}\text { La importancia de las relaciones, } \\
\text { sus valores y sobre la comunica- } \\
\text { ción con personas importantes a } \\
\text { medida que se acerca el final de la } \\
\text { vida }\end{array}$ & $\begin{array}{l}\text { Actividad lúdica con } \\
\text { rompecabezas }\end{array}$ \\
\hline
\end{tabular}

Cada sesión tenía sus objetivos:

Sesión uno:

Comprender las características existenciales

1 Esencia de la mente humana. Se utilizaron cartede los seres humanos les con historias que (sentido, libertad de voreflejan contenido eduluntad y voluntad para cativo basado en objeencontrar sentido) tivos de aprendizaje. Se crearon "canciones

Sesión dos:

Kang \& Kim $(2015)^{(6)}$
Cómo encontrar el sentido de la vida: trabajo creativo.

\section{Sesión tres:}

Cómo encontrar el sentido de la vida: en las experiencias de amor y valores.

Sesión cuatro:

Cómo encontrar el sentido de la vida: cambiar actitudes

\section{Trabajo creativo}

3 Experiencia de amor y valores

4 Actitud cambiante divertidas" para reforzar la interacción entre los participantes y los educadores y para obtener sonrisas y actitudes positivas. Se utilizaron guías educativas para el equipo de enfermería para mejorar la efectividad del Programa Centrado en el Sentido y folletos con ejercicios educativos para que los pacientes refuercen el aprendizaje al final del programa.

Sesión cinco: La importancia de la selección y la responsabilidad. 


\begin{tabular}{|c|c|c|c|c|}
\hline Artículo & Objetivos & $\begin{array}{c}N^{0} \\
\text { Sesión }\end{array}$ & Temas & $\begin{array}{c}\text { Método Educativo } \\
\text { Aplicado }\end{array}$ \\
\hline \multirow{8}{*}{$\begin{array}{l}\text { Urrego et al. } \\
(2015)^{(4)}\end{array}$} & $\begin{array}{l}\text { Comprender el concep- } \\
\text { to de Sentido da vida } \\
\text { (SV) }\end{array}$ & 1 & $\begin{array}{l}\text { Presentación } \\
\text { Explorar hechos significativos de } \\
\text { la vida. }\end{array}$ & $\begin{array}{l}\text { Relajación } \\
\text { Cuestionarios } \\
\text { Discusión }\end{array}$ \\
\hline & $\begin{array}{l}\text { Descubra la relación } \\
\text { entre hechos significa- } \\
\text { tivos en su historia de } \\
\text { vida y su contribución a } \\
\text { la construcción del SV }\end{array}$ & 2 & $\begin{array}{l}\text { Construcción de la historia colectiva } \\
\text { Identificar el impacto de ciertos } \\
\text { éxitos en la construcción de la his- } \\
\text { toria de la vida. }\end{array}$ & $\begin{array}{l}\text { Relajación } \\
\text { Reflexión } \\
\text { Discusión }\end{array}$ \\
\hline & $\begin{array}{l}\text { Reflexión sobre la in- } \\
\text { fluencia de las personas } \\
\text { en la búsqueda de SV y } \\
\text { cómo su propia historia } \\
\text { de vida puede influir en } \\
\text { la búsqueda de SV en } \\
\text { otros. }\end{array}$ & 3 & $\begin{array}{l}\text { Identificación de ciertos éxitos ex- } \\
\text { perimentados y su impacto en la } \\
\text { vida de los demás. } \\
\text { Revisión y cierre del álbum y fra- } \\
\text { ses de sesiones anteriores (el álbum } \\
\text { se inició previamente junto con el } \\
\text { terapeuta) }\end{array}$ & $\begin{array}{l}\text { Relajación } \\
\text { Reflexión } \\
\text { Revisión de tareas } \\
\text { Discusión }\end{array}$ \\
\hline & $\begin{array}{l}\text { Fortalecer los lazos } \\
\text { afectivos de los pacien- } \\
\text { tes y el reconocimiento } \\
\text { del otro como una parte } \\
\text { importante en la cons- } \\
\text { trucción de SV }\end{array}$ & 4 & $\begin{array}{l}\text { Inclusión de los acompañantes: } \\
\text { en una hoja escribir un mensaje } \\
\text { de agradecimiento y adicionar en } \\
\text { el álbum. Entrega del álbum del } \\
\text { acompañante al paciente. }\end{array}$ & $\begin{array}{l}\text { Revisión de tarea. } \\
\text { Actividad con acom- } \\
\text { pañante }\end{array}$ \\
\hline & $\begin{array}{l}\text { Diferenciar entre los } \\
\text { hechos de la vida que } \\
\text { pueden y no pueden } \\
\text { cambiarse, motivarlos a } \\
\text { actuar sobre los cuales } \\
\text { pueden tener el control, } \\
\text { como sus emociones y } \\
\text { la forma de enfrentar } \\
\text { las situaciones }\end{array}$ & 5 & $\begin{array}{l}\text { Identificación de limitaciones en la } \\
\text { vida y la existencia. } \\
\text { Presentación de video } 1 \text { (de perso- } \\
\text { najes motivadores que convirtieron } \\
\text { sus limitaciones en potencial) } \\
\text { Identificación del potencial perso- } \\
\text { nal, basado en las limitaciones iden- } \\
\text { tificadas al comienzo de la sesión. }\end{array}$ & $\begin{array}{l}\text { Relajación } \\
\text { Actividad audiovisual } \\
\text { Discusión }\end{array}$ \\
\hline & $\begin{array}{l}\text { Identifique el propósito } \\
\text { de su vida, determine } \\
\text { las responsabilidades } \\
\text { que genera y planifique } \\
\text { cómo cumplir ese pro- } \\
\text { pósito teniendo en cuen- } \\
\text { ta las limitaciones, el po- } \\
\text { tencial y la creatividad. } \\
\end{array}$ & 6 & $\begin{array}{l}\text { Identificación de objetivos y moti- } \\
\text { vaciones. } \\
\text { Modelando a partir de la historia de } \\
\text { la vida } \\
\text { Construcción colectiva de posibili- } \\
\text { dades para alcanzar objetivos indi- } \\
\text { viduales. }\end{array}$ & $\begin{array}{l}\text { Relajación } \\
\text { Actividad grupal } \\
\text { Discusión }\end{array}$ \\
\hline & $\begin{array}{l}\text { Explore el humor en } \\
\text { la vida cotidiana e in- } \\
\text { clúyalo como una her- } \\
\text { ramienta para construir } \\
\text { el sentido de la vida. } \\
\end{array}$ & 7 & $\begin{array}{l}\text { Presentación en video } 2 \text { (la impor- } \\
\text { tancia del humor en la vida diaria) }\end{array}$ & $\begin{array}{l}\text { Relajación } \\
\text { Actividad audiovisual } \\
\text { Discusión }\end{array}$ \\
\hline & $\begin{array}{l}\text { Discutir una situación } \\
\text { estresante en el pasado } \\
\text { y crear el éxito cómico } \\
\text { de la situación estre- } \\
\text { sante. }\end{array}$ & 8 & $\begin{array}{l}\text { Escribir un mensaje motivador a } \\
\text { uno de los colegas. } \\
\text { Compartiendo mensajes } \\
\text { Entrega por parte del terapeuta el } \\
\text { material escrito con los objetivos } \\
\text { de cada participante. }\end{array}$ & $\begin{array}{l}\text { Relajación } \\
\text { Doble actividad } \\
\text { Discusión grupal } \\
\text { Clausura }\end{array}$ \\
\hline
\end{tabular}

Nota: Fuente: Elaboración propia. 


\section{B. Bienestar espiritual y psicológico de la Psicoterapia Centrada en el Sentido-PCS}

El bienestar espiritual está relacionado con la disminución de las preocupaciones existenciales $^{(5,18)}$, saber cómo lidiar con la angustia ${ }^{(7)}$, aumentar los sentimientos de paz y encontrar sentido en la vida ${ }^{(8,22,21)}$. Y las mejoras psicológicas están relacionadas con una disminución de la depresión y la ansiedad, una mayor autoestima y un mayor optimismo $^{(3,5,8,19)}$.

La PCS como propuesta de intervención, visa que los pacientes tengan una mejora en su bienestar espiritual y psicológico, por lo tanto todos los estudios incluidos miden esta condición. Los siguientes cuatro estudios ${ }^{(2,4,6,18)}$ especifican sus intenciones al realizar sus investigaciones en este sentido:

Costas-Muñiz et al. (2020)(18) realizaron una adaptación cultural y lingüística de la PCS, para pacientes latinos, ya que estos representan el 18\% de la población en los Estados Unidos y era esperando 150.000 nuevos casos entre los latinos estadunidenses en 2018. Además por la ausencia de terapias para ayudar a esta población en su proceso de enfermedad, se pretendió ofrecer una psicoterapia que les diera soporte para sobrellevar su diagnóstico y mejorar el bienestar espiritual y existencial. El proceso de adaptación, fue realizado con modelos rigorosos de validez, abordando dimensiones como lenguaje, contexto, modismos, metáforas, conceptos, metas y métodos. Contaron con la participación de 70 profesionales de la salud, de dos hospitales uno en Nueva York y otro en Puerto Rico. Las recomendaciones para nuevos estudios incluye: (1) utilizar el marco de adaptación cultural para guiar las actividades de investigación y adaptación, (2) realizar investigaciones de métodos mixtos para integrar información de múltiples fuentes para el proceso de transcreación, (3) incluir un panel de expertos con diversidad lingüística y cultural, y (4) consultar a las partes interesadas, pacientes y profesionales de la salud para observar y recolectar información necesaria para modificar la intervención para responder a sus necesidades.

Otro de los estudios es de los autores Fraguell et al. $(2018)^{(2)}$ que propusieron ayudar a sus pacientes a "vivir con sentido" de una manera completa, manteniendo y expandiendo el sentido de sus vidas. Con este fin, adaptaron la PCS sigla en inglés $M C P^{(16)}$, versión grupal MCGP, para la población española y los beneficios emocionales y espirituales encontrados incluyen: crecimiento personal como resultado de su proceso de enfermedad, una mayor conciencia de su propias vidas, valorando pequeñas cosas, aceptando su diagnóstico y pronóstico, el bienestar psicológico incluye vivir con un propósito y estar conectado con otros. Las sesiones grupales de MCGP, contribuyeron para que los participantes se sintieran validados por los miembros del grupo, siendo un apoyo emocional para compartir sus propias experiencias.

A su vez, Kang y Kim (2015)(6) buscaron ayudar a pacientes jóvenes con cáncer terminal a mejorar su calidad de vida a través de una propuesta de psicoterapia llamada "El sentido de mi vida" dado que los pacientes manifestaron problemas existenciales como desesperanza, impotencia, depresión, ansiedad, falta de sentido y soledad. Las cinco sesiones de terapia desarrolladas con los pacientes tenían como objetivo comprender la existencia humana y encontrar el sentido de la vida misma. El programa utilizó estrategias educativas como la animación, el canto y actividades didácticas como la discusión, el trabajo en grupo y los ejercicios experimentales, que permitieron abordar los problemas existenciales de manera más efectiva. El principal 
efecto de este trabajo fue el aumento de la calidad de vida, que influyó positivamente en las preocupaciones existenciales de los pacientes. Los autores afirman que este tipo de intervención podría aplicarse en todas las etapas de una enfermedad potencialmente mortal y aplicarse en todos los servicios formales e informales por parte de los cuidadores que participan en los cuidados paliativos.

Asu vez, Urrego et al. (2015) $)^{(4)}$ preocupados por las crisis existenciales y espirituales que manifestaron los pacientes con enfermedades terminales, centraron su estudio en promover el bienestar espiritual, ayudar a los pacientes a buscar y construir el sentido de la vida, esto a través de revisar hechos significativos de la historia del paciente y enseñándoles cómo construir el sentido de la vida estableciendo metas y acciones para el futuro. Entre los logros alcanzados en este estudio fue fomentar la espiritualidad en términos de revelar el sentido de la vida, reducir los niveles de depresión, ansiedad, malestar psicológico y espiritual asociados con el tratamiento, así como mejorar la calidad de vida.

La PCS y sus versiones, pueden tener ingredientes únicos que son capaces de lidiar con el sufrimiento existencial en pacientes con cáncer avanzado de manera que las psicoterapias más tradicionales no guían directamente ${ }^{(8)}$. Su eficacia radica en que los pacientes experimentan el cáncer de forma adaptativa, la posibilidad de revelar el sentido de la vida a partir de la experiencia y la capacidad de transformarlo en una fuente de crecimiento personal ${ }^{(2,4)}$. A seguir se describen los modelos de PCS e su efectividad en los tipos de intervención utilizados.

\section{Terapias individuales, grupales y de pareja como estrategias eficaces de la Psi- coterapia Centrada en el Sentido-PCS}

Siete de los estudios encontrados en esta revisión, tenían como objetivo evaluar la efectividad de la PCS ${ }^{(3,5,7,8,19,21,22)}$, seis demostraron que la PCS en la versión utilizada es más relevante que otros tipos de intervenciones con pacientes y uno de los estudios al realizar una pequeña inclusión de constructos como compasión y autocompasión dentro de las sesiones de la PCS, verificaron un mayor nivel de beneficio emocional y espiritual para los pacientes ${ }^{(21)}$.

Iniciando con el estudio de Franguell, Limonero \& Gil (2020) ${ }^{(22)}$ utilizaron la modalidad de la PCS (MCP) individual versión cuidados paliativos IMCP-PC ${ }^{(23)}$, de 3 sesiones, y fue utilizada en pacientes españoles que reciben cuidados paliativos en su casa. Esta fue comparada con la psicoterapia de Counseling. Los resultados demostraron que la versión de IMCP-PC es más efectiva en el encuentro del sentido de la vida que la de Counseling. Este estudio es considerado muy relevante para la práctica clínica actual, en la medida que atiende a los pacientes desde su domicilio, ayudando aquellos que no pueden salir más de sus casas, además de comprobar que la IMCP-PC contribuyó para que los pacientes llegarán al final de la vida de una manera más serena y con menos sufrimiento. Los autores recomiendan para futuros estudios, reclutar pacientes con una mayor estimativa de vida o con más estabilidad de salud, incluso si se están acercando al final de la vida.

Otro de los estudios que realizó la PCS en la versión individual fue el de Breitbart et al. (2018) ${ }^{(19)}$ compuesto de 7 sesiones, sigla en inglés IMCP, con el objetivo de ayudar a los pacientes a reducir la angustia psicológica (depresión, ansiedad y alteraciones espirituales), mejorar la calidad de la vida y aumentar el bienestar espiritual. Fue comparado con la psicoterapia de apoyo SP y la atención habitual mejorada, sigla 
en inglés EUC. Un conjunto de pruebas fueron aplicados antes, durante y después del tratamiento ocho y dieciséis semanas después. Para medir las variables primarias usaron: SWB (bienestar espiritual), LAP-R (Índice de sentido personal) y MQOL (calidad de vida). Para las variables secundarias midieron la angustia psicológica en general, usando: HAI (evaluación de desesperanza), SAHD (actitudes hacia la muerte acelerada) y HADS-A y HADS-D (Ansiedad y depresión). La media de cambio en el bienestar espiritual y funcionamiento psicológico después del IMCP y $\mathrm{SP}$, evidenciada en todos los momentos de la aplicación de las pruebas, es mayor para IMCP que para SP. Por su vez la IMCP es un potencial de intervención adyuvante para otros tratamientos de salud mental, ya que a partir de las intervenciones se destacó una reducción de la desesperanza y depresión de los pacientes que ya estaban recibiendo otros tratamientos psicológicos. Los autores resaltan que aunque la IMCP fue diseñada para pacientes con cáncer avanzado, el enfoque y utilidad para mejorar el sentido y la calidad de vida puede tener una amplia aplicabilidad en una variedad de enfermedades y condiciones en las que las funciones físicas y psicológicas se ven afectadas adversamente ${ }^{(19)}$.

Por otro lado tenemos el estudio de Gil et al.(2018) ${ }^{(21)}$ que utilizo PCS en la versión individual para pacientes paliativos, sigla en inglés MCP-PC, incluyó 3 sesiones para pacientes hospitalizados. Los autores tomaron el modelo de la MCP-PC e incluyeron los constructos compasión y autocompasión y desarrollaron una nueva propuesta de intervención, sigla en inglés MCP-CPC, comprobando que el uso de estos dos términos durante las 3 sesiones, contribuyeron para un mayor bienestar psicológico y existencial. Esta combinación se dio a partir de los presupuestos de la terapia de la compasión, la cual posibilita al paciente comprometerse con el sufrimiento, comprender sus causas de manera no crítica y lo capacita a trabajar hábilmente para prevenir y aliviar el sufrimiento, pero los autores Gil et al. (2018)(21) percibieron que sin una conciencia plena, este proceso es limitado. Por esta razón al usar los conceptos de compasión y autocompasión con ejercicios de conexión dentro del modelo de MCP-PC, la efectividad es mayor, sin embargo los autores recomiendan realizar más investigaciones para mejorar la eficacia.

Hablando de otro estudio, los autores Breitbart et al. (2015) ${ }^{(5)}$ aplicaron en una muestra de 253 pacientes la PCS versión grupal, sigla en inglés MCGP y compararon con la psicoterapia de grupo de apoyo, sigla en inglés SGP. Se centraron en evaluar dos variables: las dependientes primarias, FACIT-Sp (Evaluación funcional del bienestar espiritual) y McGill (calidad de vida). Las variables dependientes secundarias incluyeron BECK (depresión), HADS (ansiedad), Hopelessness Assessment in Illness Questionnaire (desesperanza), MSAS (angustia de síntomas físicos) y Cronograma de actitudes en relación a la muerte acelerada. Además, se incluyeron cuatro covariables (también identificadas a priori) para controlar las posibles influencias de confusión: FSSQ (sexo, apoyo social), MMSE (funcionamiento cognitivo) y IE-12 (nivel de religiosidad) $^{(5)}$.

Los resultados de este estudio indican que: 1) Ni el sexo ni el funcionamiento cognitivo se asociaron con cambios en las variables; 2) La religiosidad, por otro lado, está significativamente relacionada con los cambios, por ejemplo, los participantes cristianos y judíos tuvieron menos sufrimiento con la enfermedad que aquellos con otra o ninguna afiliación religiosa, y 3) Aunque los participantes en ambos grupos de tratamiento han mostrado algún beneficio, la mejora fue significativamente mayor en los pacientes que recibieron MCGP que en los que no, los cambios más significativos 
son: aumento del bienestar espiritual, calidad de vida general, disminución de la depresión, ansiedad, desesperanza y síntomas físicos ${ }^{(5)}$.

El estudio de Rosenfeld et al. (2017) ${ }^{(8)}$ también objetivo investigar la efectividad de MCGP verificando hasta qué punto está se basa en el sentido de la vida (mecanismo de acción subyacente a la intervención). Para esto, realizaron proceso rigoroso con muestra aleatoria, análisis estadístico y comparación con otro enfoque de psicoterapia la SGP. Este estudio utilizó un subconjunto de medidas completadas por los participantes en la batería de evaluación: FACIT-Sp (Sentido de significado y paz y sentido de fe), McGill (calidad de vida), BECK (depresión), la falta de esperanza se midió mediante el cuestionario de desesperanza de enfermedades y el deseo de muerte prematura fue medido por el Cronograma de Actitudes en relación a la muerte adelantada.

Los resultados de este estudio destacan: 1) la principal diferencia entre las dos psicoterapias está en la mediación del MCGP que promueve la atribución del sentido de la vida y el sentimiento de paz, lo que proporcionó una mejora significativa en la calidad de vida y una disminución de la depresión, en la desesperanza y en el deseo de una muerte acelerada y 2) esta investigación no abordó en qué medida la efectividad de MCGP se debe a su mecanismo teórico.

A su vez, el estudio de Ryu et al. (2018) ${ }^{(7)}$ quería investigar la efectividad de PCS, en comparación con el grupo de control, durante y después de 2 meses. Aplicó varias escalas de evaluación que incluyeron: HADS (niveles de estrés), DS-DT (termómetro de sufrimiento), MAC (indefensa y desesperación), el Cuestionario de Calidad de Vida de la Organización Europea para la Investigación y el Tratamiento del Cáncer y QLQ-C30 (calidad de vida global) entre otras sub escalas para lograr el fenómeno estudiado.

Entre los principales resultados de este trabajo, se destacan: El grupo que participó con PCS mostró mejoras significativas en la depresión, la ansiedad y la preocupación ansiosa relacionada con el ajuste mental al cáncer, la calidad de vida global, la función cognitiva y la función social, aunque el grupo control obtuvo un puntaje relativamente alto, fue bajo para la angustia emocional y la calidad de vida en la evaluación inicial. Dos meses después de la evaluación final, se observaron mejoras para el grupo con PCS, mientras que el grupo de control mostró una tendencia a aumentar el sufrimiento, según las escalas y sub escalas utilizadas.

El séptimo artículo que se centró en la validez y efectividad de la PCS fue desarrollado por Wagner et al. (2016) ${ }^{(3)}$ quienes adaptaron esta técnica para parejas, con la justificación de que los estudios ya realizados descuidan las experiencias, necesidades y recursos construidos por los pacientes y sus cónyuges, así como reiteradas solicitudes para desarrollar intervenciones que involucren parejas, y finalmente, para evidenciar que el vínculo entre el bienestar de los pacientes y las parejas se deteriora al final de la vida.

Las evaluaciones que se llevaron a cabo, verificaron los cambios posteriores a la intervención, las escalas utilizadas fueron HADS (ansiedad y depresión), FACIT-Sp (Sentido de significado y paz y sentido de fe), CAHS (trascendencia) y $M$-VITAS (Índice de Calidad de Vida). Los principales resultados verificaron una buena viabilidad para el tratamiento y la atención espiritual de los pacientes y sus cónyuges al final de la vida. Los cambios en las variables revelan una disminución considerable en la depresión posterior a la intervención tanto para pacientes y sus parejas, por su vez la evaluación de la amenaza de los pacientes también disminuyó y la sensación 
de trascendencia aumento. Para los cónyuges, se registró una disminución de la depresión, la ansiedad y la evaluación del desafío y aumentó la sensación de paz relacionada con la enfermedad.

\section{Las limitaciones de los estudios.}

La mayoría de los estudios hacen énfasis a las limitaciones presentes en sus investigaciones, algunas que se destacan son: las muestras pequeñas dieron como resultado una potencia limitada y algunos resultados inconsistentes ${ }^{(2,5,18,21)}$. Los estudios con pacientes en estados avanzados mostraron altas tasas de desgaste durante las fases de reclutamiento, tratamiento y seguimiento, que no es inesperado para una intervención dirigida a pacientes en esta fase de la enfermedad ${ }^{(8)}$. Por su vez muestras no homogéneas de pacientes en diferentes estadios de la enfermedad puede influenciar en los resultados debido al pronóstico ${ }^{(18)}$ lo mismo para las adaptaciones culturales, los pacientes que son de una determinada región no representa la cultura general de un continente en todo su contexto ${ }^{(18)}$.

En cuanto a las intervenciones grupales, la dificultad se observó en las condiciones de salud de los pacientes y en los horarios para participar en el grupo ${ }^{(7)}$. A su vez uno de los estudios llevó a cabo intervenciones en tres hospitales y con diferentes terapeutas, lo que se observó como un factor limitante, fue que el número de pacientes que recibieron los psicólogos de cada hospital no era el mismo y que el terapeuta puede influir en el efecto terapéutico y la satisfacción del paciente ${ }^{(7)}$.

\section{Conclusiones}

De esta revisión, se puede decir que los pacientes que tienen una experiencia única y desafiante como el cáncer, manifiestan necesidades espirituales, como aumentar la esperanza, la fe, los sentimientos de paz y especialmente encontrar o fortalecer el sentido de la vida. Los estudios científicos encontrados han demostrado que, en las últimas dos décadas, los profesionales de la salud en oncología han estado dando un lugar importante para que el paciente revele el sentido de la vida, siendo una estrategia para hacerle frente a la enfermedad. Por lo tanto, con base en los resultados, se considera que la Psicoterapia Centrada en el Sentido (PCS), es una estrategia de intervención psicoterapéutica eficaz en la atención espiritual y psicológica de los pacientes con cáncer, su metodología puede ajustarse y adaptarse de acuerdo con la cultura de destino. Su efectividad se valida en los resultados de los estudios encontrados, que revelan que la experiencia del sentido de la vida en pacientes con cáncer, contribuye a la mejora de la calidad de vida, un mejor bienestar espiritual, un mejor bienestar psicológico, ajuste mental y sentimientos de satisfacción con la vida. Además de brindar beneficios significativos para los pacientes, constituye una alternativa de actuación para los profesionales de la salud y especialmente para los psicólogos, ya que la PCS apunta a una atención integral, holística y multidisciplinaria. Sin embargo todavía es poco aplicada en la región latinoamericana y con una falta de estudios desarrollados en Brasil y en el idioma portugués, lo que representa una oportunidad para su investigación y práctica. 


\section{Referencias bibliográficas}

1. Ministério da Saúde, Instituto Nacional de Câncer. (INCA). Estimativa 2020. Incidência de câncer no Brasil. Rio de Janeiro, RJ. Brasil. 2020. [Acceso 1/6/2020]. Disponible en: https://www.inca.gov.br/noticias/brasil-tera-625-mil-novos-casos-de-cancer-cada-anodo-trienio-2020-2022

2. Fraguell C, Limonero JT, Gil F. Psychological aspects of meaning-centered group psychotherapy: Spanish experience. Palliat Support Care 2018; 16: 317-24. doi: 10.1017/ S1478951517000293

3. Wagner CD, Johns S, Brown LF, Hanna N, Bigatti S. Acceptability and feasibility of a meaning-based intervention for patients with advanced cancer and their spouses: A Pilot Study. Am J Hosp Palliat Med 2016; 33: 546-54. doi: 10.1177/1049909115575709

4. Urrego BSC, Sierra MFA, Sánchez P R. Desarrollo de una intervención centrada en espiritualidad en pacientes con cáncer. Universitas Psychologica, 2015; 14(1): 299-312. [Acceso 1/6/2020]. Disponible en: http://www.scielo.org.co/scielo.php?script=sci abstract\&pid=S1657-92672015000100025

5. Breitbart W, Rosenfeld B, Pessin H, Applebaum A, Kulikowski J, Lichtenthal W G. Meaning-centered group psychotherapy: an effective intervention for improving psychological well-being in patients with advanced cancer. J Clin Oncol 2015; 33: 74954. doi: 10.1200/JCO.2014.57.2198

6. Kang KA, Kim SJ. Development and preliminary testing of a meaning-centered program for young adults with advanced-stage cancer. J Hosp Palliat Nurs 2015; 17: 213-22. doi: 10.1097/NJH.0000000000000146

7. Ryu JS, Choi SW, Yun SS, Koo BH, Choi IS, Kim SJ, Yoon DS. Preliminary findings on the effectiveness of Meaning-Centered Psychotherapy in Patients with pancreatobiliary cancer. Yonsei Med J 2018; 59: 1107-14. doi:10.3349/ymj.2018.59.9.1107

8. Rosenfeld B, Cham H, Pessin H, Breitbart W. Why is Meaning-Centered Group Psychotherapy (MCGP) effective? Enhanced sense of meaning as the mechanism of change for advanced cancer patients. Psychooncology 2017; 27: 654-60. doi: 10.1002/ pon. 4578

9. Breitbart W. Spirituality and meaning in supportive care: spirituality- and meaningcentered group psychotherapy interventions in advanced cancer. Support Care Cancer 2002; 10: 272-80. doi: 10.1007/s005200100289.

10. Medeiros AYBBV de, Pereira RE, Silva RMRCA, Rocha RCN, Moncayo GF. El sentido de la vida como recurso espiritual para el cuidado en oncología. Rev Cubana De Enf. 2019; 34(4). [Acceso 1/6/2020]. Disponible en: http://www.revenfermeria.sld.cu/index. $\mathrm{php} / \mathrm{enf} / \mathrm{article} / \mathrm{view} / 2243$

11. World Health Organization (WHO). Division of mental health and prevention of substance abuse. WHOQOL and spirituality, religiousness and personal beliefs- SRPB. Geneva: World Health Organization. 1998. [Acceso 1/6/2020]. Disponible en: http:// apps.who.int/iris/bitstream/10665/70897/1/WHO_MSA_MHP_98.2_eng.pdf

12. Frankl VE. Em busca de sentido. (Trad. de Walter Schlupp e Carlos Aveline). Petropolis: ed. Vozes 1991. [Acceso 1/6/2020]. Disponible en: http://gropius.awardspace.com/ ebooks/frankl.pdfA

13. World Health Organization. WHO. Definition of palliative Care. World Health Organization 2019. [Acceso 1/6/2020]. Disponible en: https://www.who.int/cancer/ palliative/definition/en/ 
14. National Institute for Health Research (NIHR). International prospective register of systematic reviews - PROSPERO 2019. [Acceso 1/6/2020]. Disponible en: https://www. crd.york.ac.uk/PROSPERO/

15. Moher D, Liberati A, Tetzlaff J, Altman DG, The PRISMA Group (2009) Preferred Reporting Items for Systematic Reviews and Meta-Analyses: The PRISMA Statement. PLoS Med 6(7): e1000097. doi: 10.1371/journal.pmed.1000097

16. Breitbart W, Rosenfeld B, Gibson C, Pessin H, Poppito S, Nelson C, et al. Meaningcentered group psychotherapy for patients with advanced cancer: a pilot randomized controlled trial. Psychooncology 2010; 19: 21-2. doi:10.1002/pon.1556

17. Gil FLM, Breitbart W. Psicoterapia Centrada en el Sentido: "Vivir con Sentido" Estudio Piloto. Psicooncología 2013; 10: 233-45. doi: 10.5209/rev_PSIC.2013.v10.n2-3.43446

18. Costas-Muñiz R, Torres-Blasco N, Castro-Figueroa EM, González CJ, Breitbart W, Gany F. - Meaning-Centered psychotherapy for latino patients with advanced cancer: cultural adaptation process. J Palliat Med 2020;23: 489-97 doi: 10.1089/jpm.2019.0423

19. Breitbart W, Pessin H, Rosenfeld B, Applebaum AJ, Lichtenthal WG, Li Y, Saracino $\mathrm{RM}$, et al. Individual meaning-centered psychotherapy for the treatment of psychological and existential distress: A randomized controlled trial in patients with advanced cancer. Cancer, 2018; 124:3231-9 doi: 10.1002 / cncr.31539

20. Breitbart W, Poppito S, Rosenfeld B, et al. Pilot randomized controlled trial of individual meaning-centered psychotherapy for patients with advanced cancer. J Clin Oncol 2012;30:1304-19. doi: 10.1200 / JCO.2011.36.2517

21. Gil F, Fraguell C, Benito L, Casellas-Grau A, Limonero JT. Meaning-centered psychotherapy integrated with elements of compassion: A pilot study to assess feasibility and utility. - Palliat Support Car, 2018;16: 643-7 doi: 10.1017 / S1478951518000548.

22. Fraguell HC, Limonero JT, Gil F. Psychological intervention in patients with advanced cancer at home through Individual Meaning-Centered Psychotherapy-Palliative Care: a pilot study. Support Care Cancer, 2020;10.1007/s00520-020-05322-2. doi:10.1007/ s00520-020-05322-2

23. Rosenfeld B, Saracino R, Tobias K, Masterson M, Pessin H, Applebaum A, et al. Adapting Meaning- Centered Psychotherapy for the palliative care setting: results of a pilot study. Palliat Med 2017;31:140-6. doi: 10.1177/ 0269216316651570 